\title{
FORMAÇÃO DA ESTRATÉGIA EM CONDIÇÕES DE ELEVADA INFLUÊNCIA GOVERNAMENTAL: UMA ANÁLISE LONGITUDINAL DA FUUNDACÃO DE APOIO À PESQUISA CIENTÍFICA E TECNOLÓGICA DO ESTADO DE SANTA CATARINA - FAPESC
}

\author{
Maurício Fernandes Pereira ${ }^{1}$ \\ Karla Simoni Oening ${ }^{2}$
}

\begin{abstract}
Resumo: A presente investigação analisa o processo de formação de estratégias da Fundação de Apoio à Pesquisa Científica e Tecnológica do Estado de Santa Catarina (FAPESC) - um órgão pertencente à estrutura da administração direta do Governo do Estado de Santa Catarina - com o intuito de compreender como ocorre tal construção: de forma deliberada, antecipada e racional ou de forma emergencial como conseqüência das interações entre os agentes presentes no cotidiano das organizações. Por meio de um estudo de caso de caráter longitudinal, histórico-biográfico e com respaldo nos procedimentos propostos pela Direct Research (MINTZBERG, 1979; MINTZBERG; McHUGH, 1985), resgatou-se a história da instituição no período compreendido entre os anos de 1990 e 2005. Os dados revelaram que, ao se adaptar estrategicamente, além da predominância de um comportamento estratégico planejado e sistêmico, a instituição sofreu intensa influência das políticas governamentais do Estado e que esta, associada ao baixo poder de influência sobre 0 ambiente com alto determinismo, reduziu a importância da intencionalidade gerencial no sucesso da empresa, indicando que mecanismos de seleção ambiental operam em detrimento da adaptação.
\end{abstract}

Palavras-chave: Estratégia. Mudança e adaptação. Formação da estratégia.

\section{INTRODUÇÃO}

A mudança aparece como uma das principais características do mundo contemporâneo. Ainda que inevitável e necessária à sobrevivência das instituições, sua presença restringe e reduz o tempo de planejamento e ação das organizações.

São as transformações ocorridas no ambiente as principais responsáveis pela formação de estratégias organizacionais. Se 0 ambiente muda, as organizações devem, de alguma maneira, reformular suas ações para que esse alinhamento, perdido com a mudança ambiental, seja restaurado. Além disso, percebe-se que uma crescente diversidade de grupos de interesse, os denominados stakeholders, vem exercendo forte pressão sobre os rumos estratégicos das organizações, impondo um novo padrão competitivo.

Esse conjunto de demandas sócio-ambientais - refletidas em normas, regulações, leis e inovações tecnológicas - afeta o sistema, uma vez que a legitimidade passa a ser vista como a capacidade da empresa de construir estratégias que integrem, de maneira sinérgica, aspectos econômicos, ambientais e sociais, contemplando satisfatoriamente as demandas dos múltiplos atores, que interagem em um ambiente institucional cada vez mais controvertido.

\footnotetext{
1 Programa de Mestrado em Administração; UFSC, Florianópolis, SC. E-mail: $\underline{\text { mpereira@ @cse.ufsc.br }}$

2 Mestre em Administração, UFSC, Florianópolis, Estado. E-mail: koening @ eletrosul.gov.br
} 
As Fundações de Apoio à Pesquisa, categoria na qual se enquadra a instituição estudada, por sua natureza singular, revelam-se mais suscetíveis ao ambiente em que estão inseridas, uma vez que, por estarem sob a administração direta do Governo do Estado de Santa Catarina, devem refletir os anseios da sociedade.

Assim, a compreensão dos processos que originam as estratégias pode representar a descoberta de uma rota capaz de conduzir as organizações à sobrevivência e ao sucesso. Portanto, desvendar como as estratégias surgem e são implementadas permite que a administração se prepare para lidar com o contexto no qual emergem tais estratégias.

Nessa perspectiva, o presente artigo tem o propósito de verificar empiricamente o processo de formação de estratégias na Fundação de Apoio à Pesquisa Científica e Tecnológica do Estado de Santa Catarina (FAPESC) no período compreendido entre 1990 e 2005, considerando quais foram e como se desenvolveram as mudanças, bem como os fatores motivaram a organização a empreendêlas.

\section{REVISÃO BIBLIOGRÁFICA}

Delineia-se nesta seção, um breve arcabouço conceitual da pesquisa, abordando os elementos fundamentais para a compreensão do processo de formação da estratégia. São discutidos os conceitos de estratégia e os modelos teóricos de seu processo de formação, os quais contribuirão para o alcance do objetivo da investigação.

\subsection{ESTRATÉGIAS: UMA VISÃO GERAL}

O conceito de estratégia não encontra unanimidade na literatura. Desde a sua origem até sua popularização, nas décadas de 60 e 70, o conceito passou por uma sucessão de redefinições. Embora estas concordem que a estratégia representa um elo entre a organização e 0 ambiente, a discussão sobre a formulação e implementação das mesmas é extensa.

Enquanto alguns autores compreendem a estratégia como um conjunto de atividades indivisíveis e simultâneas, resultantes de um processo dinâmico e emergente, decorrente das interações entre os agentes presentes no cotidiano das organizações (MINTZBERG; AHLSTRAND; LAMPEL, 2000; QUINN, 1980), outros evidenciam seu caráter prescritivo, intencional e racional, no qual existe uma clara desarticulação entre o planejamento e a ação, ou seja, a estratégia deve ser primeiro formulada para, posteriormente, ser implementada (ANSOFF, 1965; STEINER, 1969; CHANDLER, 1962; ANDREWS, 1971).

$\mathrm{Na}$ concepção prescritiva, a estratégia é entendida como um conjunto de cursos de ação, linhas-mestras concebidas de forma consciente para lidar com determinada situação (COSTA; SILVA, 2002). Ansoff (1965) corrobora essa idéia, argumentando que a estratégia deve ser planejada em um processo de análise formalizado e controlado, que resulte na definição de planos de ação para atingir objetivos predefinidos. Esses planos, de acordo com Steiner (1969), devem ser objetivos, factuais, lógicos e realistas - ao máximo possível - para fixar objetivos e arquitetar meios de obtê-los.

Essa forma de conceber a estratégia estaria, conforme Mintzberg (2004), ligada à tradição militar de distinguir estratégias de táticas, decompondo e determinando a importância de cada uma das 
atividades a priori. As estratégias dizem respeito às ações importantes, ao passo que as táticas relacionam-se aos simples detalhes.

Para Mintzberg (1973), no entanto, esse entendimento é restrito e ignora o fato de a estratégia ser, acima de tudo, um processo de aprendizado de padrões, os quais se desenvolvem a partir do comportamento das pessoas e do que elas aprendem ao longo do caminho. Os estudos de Lindblom (1959) foram pioneiros no reconhecimento de que a definição dos objetivos ocorre quase que ao mesmo tempo em que as ações são deflagradas. Foram esses estudos que serviram de inspiração para 0 que Mintzberg (2000) chamou de estratégia emergente. Na compreensão desse autor, a formação da estratégia apóia-se em dois alicerces fundamentais: um deliberado e outro emergente. Esses dois processos formam os extremos de um continuum, ao longo do qual as estratégias criadas no mundo ideal e no mundo real podem ser visualizadas em algum ponto do traçado (MINTZBERG; QUINN, 2001).

A principal característica da estratégia emergente é de que a organização é capaz de responder ao ambiente e de interagir com ele de uma maneira desestruturada e flexível, sem um curso de ação ou plano previamente estruturado. Uma idéia leva a outra até que um novo padrão seja formado. A ação precedeu, portanto, a racionalidade (MINTZBERG, 1987).

$\mathrm{O}$ que ocorre na prática é que abordagens bem sucedidas transformam-se em um padrão de ação que, conseqüentemente, tornam-se estratégias. Dessa forma, alguns planos podem não ser realizados, assim como podem surgir padrões que não tenham sido preconcebidos, caracterizando 0 que Mintzberg e McHugh (1985) denominam estratégias emergentes: padrões observados de ações não formulados formal ou previamente, conforme pode ser observado na Figura 01. Em resumo, podese perceber que planos completamente efetivados podem ser chamados de estratégias deliberadas, enquanto as estratégias emergentes resultam de padrões observados, não formalmente pretendidos, em ações que se desenvolveram uma a uma, convergindo, ao longo do tempo, em algum tipo de padrão.

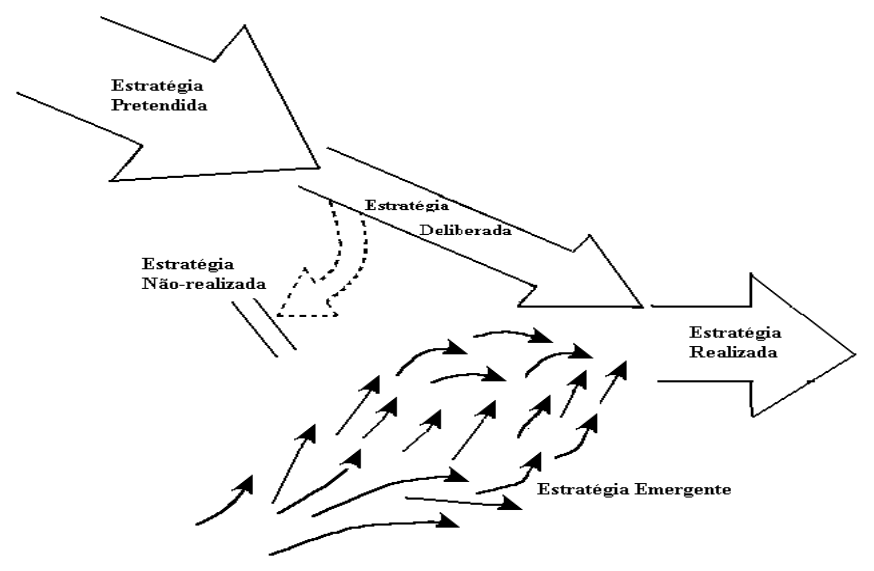

\section{Figura 1 - Estratégia Deliberada versus Estratégia Emergente}

Fonte: Mintzberg (1998)

Quinn (1980) também vem contribuindo com a idéia de estratégia emergente. Seus estudos revelam as limitações do processo formal de planejamento, o qual teria um grau de utilidade restrito em função da distância entre o processo de planejamento e os problemas vivenciados pelas organizações. Para 0 autor, nas grandes empresas, as estratégias mais efetivas tendem a emergir aos poucos, em um processo razoavelmente amplo e interativo. Desse modo, a organização aprenderia e assimilaria 
informações e mudanças a partir de uma série de comprometimentos parciais localizados e não a partir de formulações globais de estratégias definitivas. A essa série de comprometimentos pontuais, isolados, que gera um padrão coerente, ele denominou de "incrementalismo lógico".

Hardy e Fachin (1996) apresentam nove tipos de estratégias que podem ser adotadas pelos atores dominantes das instituições para lograr a consecução de seus projetos: planejada, empreendedora, ideológica, guarda-chuva, processual, desconexa, consensual, imposta e nãorealizada. As características principais de cada tipo de estratégia estão descritas no Quadro 01.

\begin{tabular}{|l|l|}
\hline \multicolumn{1}{|c|}{ TIPOS DE ESTRATÉGIAS } & \multicolumn{1}{c|}{ CARACTERÍSTICAS } \\
\hline Estratégia planejada & $\begin{array}{l}\text { Assemelha-se à noção de estratégia deliberada. Consiste em intenções precisas, } \\
\text { formuladas e articuladas pela liderança principal da organização. }\end{array}$ \\
\hline Estratégia empreendedora & $\begin{array}{l}\text { É relativamente deliberada, porém, por não ser explícita, existe a possibilidade do líder } \\
\text { mudá-las rapidamente e, assim, as estratégias podem emergir. }\end{array}$ \\
\hline Estratégia ideológica & $\begin{array}{l}\text { Existe como uma forma coletiva de todos os atores organizacionais. São relativamente } \\
\text { difíceis de mudar em virtude de crenças compartilhadas e do controle que se firma na } \\
\text { socialização de seus membros. }\end{array}$ \\
\hline Estratégia guarda-chuva & $\begin{array}{l}\text { Consiste em metas amplamente definidas pela liderança, permitindo aos outros atores a } \\
\text { decisão sobre como melhor alcançá-las. Assim, o objetivo maior é deliberado, mas o } \\
\text { caminho em direção ao objetivo, emerge. }\end{array}$ \\
\hline Estratégia processual & $\begin{array}{l}\text { Ocorre quando a liderança estabelece termos de referência de modo a criar condições mais } \\
\text { prováveis para que os resultados pretendidos surjam. }\end{array}$ \\
\hline Estratégia desconexa & $\begin{array}{l}\text { Ocorre em partes distintas da organização, podem ser deliberadas ou emergentes, dentro } \\
\text { de cada unidade específica. }\end{array}$ \\
\hline Estratégia consensual & $\begin{array}{l}\text { É negociada entre os membros ou é formada por ajustamento mútuo entre os mesmos, } \\
\text { com ausência de diretivas centrais. }\end{array}$ \\
\hline Estratégia imposta & $\begin{array}{l}\text { Refere-se à imposição de diretivas estratégicas sobre a organização por forças externas à } \\
\text { mesma. }\end{array}$ \\
\hline Estratégia não-realizada & É a estratégia pretendida que não consegue se materializar em termos de ações efetivas. \\
\hline
\end{tabular}

\section{Quadro 1 - Tipologia de estratégias}

Fonte: Hardy; Fachin (1996)

Whittington (2002) oferece uma forma ampla de conceituar a estratégia, propondo quatro abordagens genéricas para a sua conceituação - clássica, evolucionária, contextualista e sistêmica (Figura 02) - as quais contemplam os conceitos de estratégia abordados na literatura.

Para a abordagem clássica, a estratégia constitui-se de um processo racional de cálculo e de análises deliberadas, com objetivo de maximizar as vantagens no longo prazo. Para a abordagem evolucionária, a estratégia no sentido clássico do planejamento racional é irrelevante, uma vez que 0 ambiente é muito irregular e volátil para que sejam feitas previsões eficazes.

Essas quatro abordagens se distinguem fundamentalmente em duas dimensões: os resultados da estratégia e os processos pelos quais ela é concebida. As abordagens clássica e evolucionária vêem a maximização do lucro como resultado natural do desenvolvimento da estratégia. As abordagens sistêmica e processual são mais pluralistas, percebendo outros resultados possíveis, além do lucro. As abordagens também se diferenciam com respeito aos processos. A evolucionária se alia à processualista ao enxergar a estratégia como algo que emerge dos processos casuais, confusos e conservadores. Por outro lado, embora diferentes quanto a resultados, as abordagens clássica e sistêmica concordam que a estratégia pode ser algo deliberado (WHITTINGTON, 2002). 


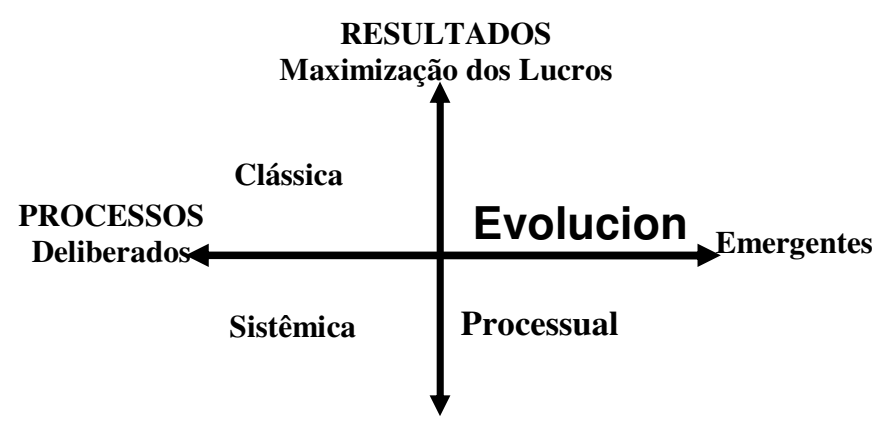

Figura 2 - Perspectivas genéricas sobre estratégia

Fonte: Whittington (2002)

\subsection{O AMBIENTE ORGANIZACIONAL: A INFLUÊNCIA DOS STAKEHOLDERS NA FORMAÇÃO DA ESTRATÉGIA}

As organizações participam de ambientes nos quais realizam operações que Ihes possibilitam a sobrevivência e o desenvolvimento. Com a rápida ascensão dos relacionamentos cooperativos, a formação de estratégias abandona os limites da organização isolada para se tornar um processo conjunto a ser desenvolvido com parceiros. Essa abordagem teórica parte do pressuposto de que as organizações não operam no abstrato e que não podem ser entendidas apenas do ponto de vista dos líderes. Assim, o desenvolvimento de estratégias, relacionadas ao contexto ambiental, requer a interação das empresas com os vários stakeholders que as influenciam ou são influenciados por elas.

A compreensão do ambiente organizacional passa, necessariamente, pela decodificação da relação existente entre as organizações e seus stakeholders, ou seja, os diversos grupos afetados por suas operações ou que, de alguma forma, as influenciam como funcionários, clientes, fornecedores, comunidade em geral, entidades governamentais e tantos outros (BOWDITCH; BUONO, 1997).

A abordagem teórica dos stakeholders propõe-se, então, a procurar resposta para algumas questões de cunho operacional, quais sejam: qual o papel de cada um dos stakeholders na formação da estratégia na instituição? que tipo de influência tem sobre a empresa? quais devem ser levados em consideração no processo de formação de estratégias? quais dos interesses devem ser privilegiados para assegurar a sustentabilidade da empresa no longo prazo?

\subsection{O PROCESSO DE FORMAÇÃO DE ESTRATÉGIA}

Frequentemente, o processo de formulação de estratégias é confundido com o processo de formação. 0 processo de formulação é prescritivo, as estratégias são consciente e explicitamente construídas. Já o processo de formação é descritivo, as estratégias são inconsciente e implicitamente construídas.

Os processos que dão origem as estratégias têm influência direta sobre as definições da mesma. A forma como a estratégia surge e é implementada não é idêntica em todas as organizações, 
resultando de uma conjugação de fatores externos (características e condições do meio ambiente) e de condições internas (capacidades da organização) que configuram cada situação em particular.

0 processo estratégico está relacionado à maneira como se concebe a estratégia: como perspectiva de futuro na qual as decisões são formuladas antecipadamente (o que se pretende fazer), ou, ao contrário, pela perspectiva do padrão das ações que a organização ao longo do tempo assume (o que foi feito). Assim, a compreensão dos processos que originam as estratégias é tão importante quanto 0 entendimento das definições de estratégia.

Nicolau (2001) apresenta três propostas para orientar a análise da formulação de estratégias organizacionais: processo racional-formal, processo negociado e processo em construção permanente. Essas abordagens não podem ser consideradas como alternativas que se excluam mutuamente. $\mathrm{Na}$ prática, a formulação da estratégia organizacional pode, de forma simultânea, incluir os três procedimentos, ou seja, o processo pode envolver a racionalização, o planejamento formal, a negociação com atores internos e externos que o torne efetivo e, ainda, ser o suficientemente flexível para não desprezar o processo de aprendizagem gerado pelos agentes, a partir da interpretação que os mesmos fazem de situações que emergem do ambiente interno e externo à organização. Percebese que, enquanto os dois primeiros modelos caracterizam-se por comportarem etapas seqüenciais, 0 terceiro traz como aspecto-chave a simultaneidade da ocorrência das etapas. Por isso, esse último pode ser considerado com um processo de formação de estratégias.

Como um processo racional-formal, a estratégia se desenvolve por meio de uma série de etapas seqüenciais, racionais e analíticas, que envolvem um conjunto de critérios objetivos. Esses critérios são baseados na racionalidade econômica que auxilia os gestores na análise das alternativas estratégicas e na tomada de decisão.

A formação de estratégia como um processo negociado tem - nos sistemas de valores internos e externos, no meio-ambiente e nas capacidades e recursos da organização - os fatores determinantes e condicionantes para a escolha final. Esse enfoque valoriza o processo de negociação como um dos aspectos mais importantes a considerar na formação da estratégia e valoriza o papel dos atores intervenientes, acentuando a importância da dimensão social e política no processo.

A formação de estratégia como um processo em construção permanente, por sua vez, destaca a complexidade das estratégias. A formação da estratégia é um processo complexo, no qual as perspectivas de racionalidade e de negociação não contemplam todas as dimensões inerentes ao processo de formulação de estratégia. O sistema de planejamento formal centra-se em fatores quantitativos, desvalorizando os aspectos qualitativos (comportamentais e organizacionais), já a abordagem comportamental se fixa nas relações psicossociológicas de poder e nos comportamentos. Há situações, no entanto, em que o contexto ambiental e organizacional passa por mudanças rápidas e abruptas, no qual nem um processo - racional ou negociado - é capaz de oferecer condições para a formação de estratégias efetivas. 0 processo vai se formando pela aprendizagem sobre 0 meio ambiente, pelas capacidades internas da organização e pela forma como se estabelece a relação entre esses dois eixos. A estratégia tenderá a ser desenvolvida como decorrência das ações que vão se desencadeando, cujas implicações para 0 futuro são impossíveis de serem conhecidas antecipadamente.

Para classificar as diferentes abordagens na formulação da estratégia, Idenburg (1993) utiliza duas dimensões: orientação para objetivos (o quê) e orientação para processos (como). Delas derivam quatro visões do processo de desenvolvimento da estratégia, constantes na Figura 03: Planejamento Racional, Incrementalismo Lógico, Estratégia Emergente e Aprendizagem Orientada. 


\begin{tabular}{|c|c|c|c|}
\hline \multirow{3}{*}{$\begin{array}{l}\text { ORIENTAÇÃO PARA } \\
\text { PROCESSOS } \\
\text { (como) }\end{array}$} & & FORTE & FRACO \\
\hline & FORTE & $\begin{array}{l}\text { Incrementalismo } \\
\text { Lógico }\end{array}$ & $\begin{array}{l}\text { Aprendizagem } \\
\text { Orientada }\end{array}$ \\
\hline & FRACO & $\begin{array}{l}\text { Planejamento } \\
\text { Formal }\end{array}$ & $\begin{array}{l}\text { Estratégia } \\
\text { Emergente }\end{array}$ \\
\hline
\end{tabular}

\section{Figura 3 - Visões do processo de desenvolvimento da estratégia}

Fonte: Idenburg (1993)

A abordagem do Planejamento Racional, que tem como principais autores Porter (1991) e Ansoff (1991), baseia-se na hipótese de um ambiente previsível, de forma que a futura posição da organização pode ser determinada em termos de objetivos quantificados. O processo tem início com a formulação de objetivos viáveis e, a partir daí, são utilizados argumentos racionais e analíticos como ferramentas para otimizar soluções para problemas definidos, os quais conduzem a um plano para a ação. Para as definições das estratégias, são analisadas as oportunidades e as ameaças do ambiente externo e as forças e fraquezas do âmbito interno à organização. A formulação do plano é distinta de sua implementação: planeja-se primeiro o que se vai implementar depois.

Para a abordagem do Incrementalismo Lógico, formulada por Quinn (1980), o processo bem sucedido de desenvolvimento da estratégia abrange, concomitantemente, elementos de orientação para objetivos e de orientação para processos. O processo desenvolve-se em fases, cada qual apoiada na anterior e tem sua lógica interna: a estrutura acompanha a estratégia, entretanto a estrutura organizacional também impulsiona 0 desenvolvimento da estratégia. A gestão estratégica não se sustenta apenas na racionalidade, mas também em emoções, dúvidas, desejos e intuição.

A abordagem da Estratégia Emergente, proposta por Mintzberg (1987), defende que não é possível desenvolver a perspectiva do futuro e formular objetivos explícitos em um ambiente imprevisível; ao contrário, é necessário reagir de forma flexível, oportuna e acidental às pressões do ambiente.

A aprendizagem Orientada é bastante voltada aos processos e pouco direcionada aos objetivos. Essa perspectiva, defendida por De Geus (1988) e Senge (1999), parte da premissa de que a simples formulação de planos estratégicos não assegura as transformações comportamentais dos administradores, pois, em geral, seus modelos mentais não consideram a complexidade do mundo. De acordo com essa abordagem, uma imagem comum da realidade, uma linguagem comum e a aquisição conjunta de novos insigths são tão importantes quanto a definição de objetivos precisos para o futuro pretendido. Dentro desse quadro, são utilizados cenários, não por sua qualidade prescritiva ou orientação para objetivos, mas para influenciar conscientemente os modelos mentais e a linguagem comum. $O$ desenvolvimento da estratégia é, portanto, um processo permanente de melhorias contínuas.

Mintzberg (1973) descreve três modos ou categorias de processos de formação de estratégia:

a) Modo empreendedor - está baseado nas características do empreendedor e apresenta quatro elementos principais: a estratégia dominada pela busca de novas oportunidades; 0 poder centralizado nas mãos do executivo principal; a estratégia formulada por meio de grandes e arrojados saltos e o crescimento que consiste no objetivo principal da 
organização empreendedora. Isso significa que, ao operar no modo empreendedor, a organização entende que 0 ambiente como algo maleável, como uma força que pode ser confrontada e controlada.

b) Modo adaptativo - caracteriza-se por soluções reativas a problemas existentes por meio de passos incrementais. Quatro grandes características distinguem o modo adaptativo de formação de estratégias: não existem objetivos claros na organização, ou seja, a formulação de estratégia reflete uma divisão de poder entre os membros de uma coalizão complexa, que prende a organização em uma teia de forças políticas; o processo é caracterizado pela solução por reação aos problemas existentes ao invés da busca de novas oportunidades; a organização toma decisões em passos incrementais e consecutivos; a formulação de estratégias é fragmentada e o formulador no modo adaptativo se mantém flexível e livre para se adaptar às necessidades do momento.

c) Modo planejado - baseia-se na análise de propostas e cenários, gerando estratégias globais para a organização. Três características principais delineiam o modo planejado: 0 analista desempenha papel importante na formulação da estratégia; há concentração de esforços na análise sistemática, particularmente na avaliação de custos e benefícios das propostas correntes; a integração de decisões e estratégias é uma característica-chave.

As condições que levam uma organização a adotar preponderantemente um modo em vez de outro, relaciona-se com as características da própria organização, tais como: tamanho, natureza de sua liderança e aspectos de seu ambiente, tais como competição e estabilidade.

\section{ASPECTOS METODOLÓGICOS}

O propósito principal deste trabalho é conhecer o processo de formação das estratégias da Fundação de Apoio à Pesquisa Científica e Tecnológica do Estado de Santa Catarina (FAPESC), determinando quais foram, como se desenvolveram e que fatores ocasionaram as mudanças estratégicas ocorridas na instituição desde sua fundação, no ano de 1990, até 0 ano de 2005.

Para alcançar esse objetivo, a pesquisa foi desenvolvida por meio de um estudo de caso singular (YIN, 1990), com fundamento em uma abordagem qualitativa do tipo interpretativa (GODOY, 1995; RICHARDSON et al, 1985). Optou-se por essa metodologia porque a proposta de estudar o processo de formação de estratégias por um período de tempo envolve uma carga histórica, cultural, política e ideológica que não pode ser simplificada em uma fórmula numérica ou em um dado estatístico (MINAYO, 1994).

Adotou-se uma metodologia contextualista e histórico-biográfica (KIMBERLY, 1976; SALAMA, 1994; VAN DE VEN; POOLE 1992), abalizada na análise processual e contextual das mudanças estratégicas ocorridas na FAPESC, identificando seus antecedentes e suas conseqüências ao longo do tempo. Para orientar a investigação, foram consideradas as dimensões propostas por Pettigrew (1985a; 1985b), analisando a mudança em termos de conteúdo (o que mudou), contexto (porque mudou) e processo (como mudou).

Como método de coleta e análise dos dados, foram utilizados os procedimentos propostos pela Direct Research, concebida por Mintzberg (1979) e Mintzberg e McHugh (1985), fazendo uma 
reconstrução histórica do processo de formação estratégica ao longo do ciclo de vida da FAPESC. Foram cumpridas as seguintes etapas:

a) Coleta de dados secundários em publicações, atas das reuniões de Conselho e das reuniões de Diretoria, correspondências e documentos internos.

b) Arranjo dos dados coletados secundários em ordem cronológica, agrupando-os em períodos com características semelhantes.

c) Coleta de dados primários por meio de entrevistas semi-estruturadas com membros da administração superior da Fundação.

d) Construção de uma lista de eventos que influenciaram decisivamente na formação da estratégia da instituição, como sugerido por Miles e Huberman (1984) e Mintzberg e McHugh (1985), com base nos dados provenientes da pesquisa documental e das entrevistas, enfatizando os períodos de crise. Com a descrição desses eventos, procurouse reconstruir e analisar historicamente a formação das estratégias na FAPESC.

e) Definição dos períodos estratégicos, com base na interpretação dos pesquisados e do pesquisador.

f) Análise teórica de cada período: comparou-se as entrevistas realizadas aos dados secundários, buscando correlações entre estes e a teoria utilizada.

\section{ANÁLISE E DISCUSSÃO DOS RESULTADOS}

\subsection{O CARÁTER ATÍPICO DA INSTITUIÇÃO EM ANÁLISE}

A FAPESC pode ser considerada uma organização complexa e singular, pois, apesar de exibir as mesmas características e necessidades das demais, seus processos e demandas diferenciam-se, distinguindo-a de organizações industriais, empresas de serviços e até mesmo de outros órgãos governamentais. Suas características não podem ser rotuladas de "burocráticas", uma vez que tal denominação traz, em seu bojo, a conotação de estabilidade ou até mesmo rigidez e essas organizações são mais fluidas.

O estudo inclui a pesquisa de duas instituições que antecederam a FAPESC: o Fundo Rotativo de Fomento à Pesquisa Científica e Tecnológica (FUNCITEC), denominação utilizada na ocasião de sua criação, no ano de 1990, e que permaneceu até o ano de 1997, quando passou a chamar-se Fundação de Ciência e Tecnologia, mantendo a mesma sigla e denominação até 2005.

Integrante do Sistema Estadual de Ciência e Tecnologia do Estado de Santa Catarina, a FAPESC é responsável, em conjunto com a Secretaria de Estado da Educação, Ciência e Tecnologia, pelo fomento à Ciência e Tecnologia no âmbito estadual. A FAPESC foi criada pela Lei Complementar no 284, de 28/02/2005, absorvendo, também, o Fundo Rotativo de Estímulo à Pesquisa Agropecuária do Estado de Santa Catarina (FEPA).

Destacam-se como características da FAPESC: 
a) clientela especial: o leque de clientes é amplo e estes participam ativamente do seu processo decisório;

b) profissionalismo: a maioria do corpo funcional é composta por funcionários públicos e coordenadores de projetos, exercendo funções não rotineiras que demandam autonomia;

c) vulnerabilidade ambiental: por fazer parte da estrutura hierárquica do Governo Estadual, o caráter social nas suas ações não pode ser abandonado, assim, a forte influência do ambiente externo pode afetar os processos organizacionais de gestão;

d) a alteração da estrutura de poder obedece às mudanças políticas do Governo do Estado, o que dificulta a continuidade das ações da instituição quando existe mudança de Governo especialmente se essa mudança envolve troca partidária;

e) as considerações políticas tendem a dominar e muitos grupos de interesse influenciam 0 processo decisório;

f) a estrutura de poder é formalmente definida em legislação, no entanto, existe indefinição quanto aos papéis;

g) as mudanças geralmente ocorrem como imposição das decisões advindas do Governo do Estado, da Assembléia Legislativa e dos clientes;

h) a tomada de decisão é incrementalista e envolve a participação da Diretoria, das instituições de ensino e pesquisa do Estado e dos representantes empresariais, por meio de deliberação do Conselho de Administração;

i) não existe padrão de performance e de compromissos com resultado e há dificuldade de se mensurar os produtos resultantes da ação organizacional.

O elenco dessas características evidencia que a instituição possui características atípicas e distintas das demais organizações empresariais, merecendo, portanto, estudo diferenciado.

\subsection{O PROCESSO DE FORMAÇÃO DA ESTRATÉGIA NA FAPESC}

Várias estratégias se formaram ao longo do período abordado por este estudo longitudinal (1990-2005). Para explicar a formação das estratégias, foram destacados os eventos críticos que influenciaram decisivamente a trajetória do mesmo, servindo-se de três critérios já utilizados por Cunha (1996), quais sejam: ênfase conferida ao tema pelos entrevistados, suas percepções pelas conseqüências dos eventos e a relevância destes no processo de adaptação estratégica da instituição. Esses eventos, por sua vez, foram reunidos em períodos específicos do processo de adaptação estratégica, os denominados períodos estratégicos. O período estratégico é considerado como um conjunto de decisões que suscita um padrão de comportamento estratégico característico do período.

$\mathrm{Na}$ investigação do processo de adaptação da FAPESC, foram identificados cinco períodos estratégicos (Tabela 01) que serão arcabouço para a identificação das estratégias formadas na FAPESC ao longo da sua existência. 


\begin{tabular}{|l|c|}
\hline \multicolumn{1}{|c|}{ DESCRIÇÃO } & PERÍODO \\
\hline $\begin{array}{l}\text { Criação e estruturação do Sistema de Ciência e Tecnologia do Estado de Santa Catarina: os primeiros anos } \\
\text { do Fundo Rotativo de Fomento à Pesquisa Científica e Tecnológica do Estado de Santa Catarina }\end{array}$ & 1990 -1996 \\
\hline Criação da Fundação de Ciência e Tecnologia (FUNCITEC) estruturação organizacional e crise financeira & 1997 \\
\hline Equacionamento da dívida e ênfase na inclusão digital/social & 1999 - 2002 \\
\hline Foco na ampliação das modalidades de fomento e na regionalização dos recursos & 2003 - 2004 \\
\hline Reestruturação, incorporação e crescimento & 2005 \\
\hline
\end{tabular}

\section{Quadro 2 - Períodos estratégicos identificados durante a adaptação estratégica da FAPESC} (1990-2005)

Fonte: Dados primários

4.2.1 Período estratégico I: Criação e estruturação do Sistema de Ciência e Tecnologia do Estado de Santa Catarina: os primeiros anos do Fundo Rotativo de Fomento à Pesquisa Científica e Tecnológica do Estado de Santa Catarina (FUNCITEC)

Registra-se o surgimento do Fundo Rotativo de Fomento à Pesquisa Científica e Tecnológica do Estado de Santa Catarina, o FUNCITEC, que precede a Fundação de Ciência e Tecnologia, com a mesma sigla, e a atual Fundação de Apoio à Pesquisa Científica e Tecnológica do Estado de Santa Catarina, a FAPESC. Dois eventos, em particular, contribuíram para a instituição do FUNCITEC:

- a promulgação da Constituição Federal, no ano de 1988, que facultou aos Estados a destinar parcela de sua receita orçamentária a entidades públicas de fomento, ao ensino, à pesquisa científica e tecnológica;

- a promulgação da Constituição do Estado de Santa Catarina, no ano seguinte, que tornou obrigatória a destinação de parcela de suas receitas para a pesquisa científica e tecnológica.

Nesse período, a FUNCITEC não conseguiu se estruturar administrativa e financeiramente para os objetivos aos quais se destinava. Seu alcance, no âmbito estadual, foi reduzido e os investimentos também foram pouco significativos, pautaram-se em apenas dois programas: a Rede Catarinense de Ciência e Tecnologia e o Prêmio Mérito Universitário, ambos propostos pelo Governo do Estado. Ao longo dos seis anos que compõem o primeiro período estratégico, foram identificados seis eventos críticos que influenciaram o processo de adaptação estratégica da instituição, são eles:

- a criação do Fundo Rotativo de Amparo à Pesquisa Científica e Tecnológica do Estado de Santa Catarina (FUNCITEC) e a estruturação do Sistema Estadual de Ciência e Tecnologia, em 1997;

- o Fundo Rotativo de Amparo à Pesquisa Científica e Tecnológica do Estado de Santa Catarina (FUNCITEC) passa a ser coordenado pela Secretaria de Estado de Tecnologia, Energia e Meio Ambiente, em 1991;

- a criação do Programa Prêmio Mérito Universitário Catarinense, em 1994;

- a eleição de Paulo Afonso Evangelista Vieira para Governador do Estado, em 1995; 
- a gestão do Fundo Rotativo de Amparo à Pesquisa Científica e Tecnológica do Estado de Santa Catarina (FUNCITEC) passa a ser responsabilidade da Secretaria de Estado do Desenvolvimento Econômico, Científico e Tecnológico, em 1995;

- a criação da Rede Catarinense de Ciência e Tecnologia (RCT), em 1995.

A análise dos eventos permite destacar o fundo de fomento aos programas de Ciência e tecnologia e as ações de fomento ao desenvolvimento empresarial do Estado como estratégias organizacionais relevantes da FUNCITEC. Pois, naquele momento, o foco estava no desenvolvimento científico e tecnológico das empresas e universidades instaladas no Estado, visando o destaque deste frente ao cenário nacional.

Sob a ótica de Whittington (2002), ambas são classificadas como Estratégias Sistêmicas. Primeiro, por surgirem de processos deliberados, pois tanto a criação do Fundo quanto os programas por ele apoiados foram aprovados em Lei. Segundo, porque nenhuma das estratégias buscava a maximização do lucro. Ao contrário, por se tratar de um programa de governo, a questão social esteve sempre presente. Até mesmo ao atuar como agente financiador das atividades empresariais, a finalidade mais premente era fortalecer a economia, gerar empregos etc.

\subsubsection{Período estratégico II: Criação da Fundação de Ciência e Tecnologia (FUNCITEC): estruturação organizacional e crise financeira}

No final de 1996, a partir da Reforma Administrativa que instituiu o Programa de Modernização Tecnológica para o Estado de Santa Catarina (PROMOTEC/SC), o então Governador Paulo Afonso propôs, à Assembléia Legislativa, a criação de uma fundação destinada ao fomento da ciência e tecnologia como órgão central do Sistema de Ciência e Tecnologia do Estado. A instituição - pela Lei 10.355, de 09/01/1997 - da Fundação de Ciência e Tecnologia (FUNCITEC) assinala o início do segundo período estratégico identificado nesta investigação. No período que abarcou o ano de 1997, foram identificados quatro eventos críticos que afetaram o processo de adaptação estratégica da Fundação, quais sejam:

- a instituição da Fundação de Ciência e Tecnologia (FUNCITEC);

- o lançamento do Edital Sul de Pesquisa e Pós-Graduação;

- a gestão do Parque Alfa passa a ser responsabilidade da FUNCITEC

- a crise financeira da FUNCITEC devido aos reflexos do Escândalo das Letras.

Foram adotadas como estratégias do período: a atuação no estímulo a empresas de base tecnológica e na capacitação de pessoal, assim como na melhoria da qualidade das instituições de ensino e pesquisa do Estado; a operação como agência oficial de fomento da pesquisa Científica e Tecnológica no Estado de Santa Catarina e a atuação como financiadora dos Programas do Governo e na criação de um Sistema Estadual de Informação em Ciência e Tecnologia.

De acordo com a classificação proposta por Whittington (2002), as estratégias foram Sistêmicas, pois, além de planejadas (previstas no Decreto de criação da FUNCITEC e no Plano Plurianual), almejavam mais que a maximização dos lucros, sua finalidade principal pautava-se no desenvolvimento científico das instituições de ensino e pesquisa e, acima de tudo, em tornar o Estado competitivo no âmbito nacional e internacional. 


\subsubsection{Período estratégico III: Equacionamento da dívida e ênfase na inclusão digital/social}

Com o encerramento do mandato do Governador Paulo Afonso, o Governo do Estado é assumido por um político da aliança partidária adversária, Esperidião Amin, que empossa a nova Administração Superior da FUNCITEC e indica Honorato Tomelin para a função de Diretor Geral. Esse fato marcou o início do terceiro período estratégico da instituição. Nesse período estratégico, 1999 a 2002, quatro eventos influenciaram, de maneira significativa, o processo de adaptação estratégica e de mudança na instituição pesquisada:

- mudança na estrutura de poder da FUNCITEC: Honorato Tomelin assume a Direção Geral e o Conselho Superior assume efetivamente suas atribuições de órgão de deliberação máxima da FUNCITEC, em 1999;

- elaboração do $1^{\circ}$ planejamento formal das atividades da instituição, em 2000;

- formulação e lançamento de $1^{\circ}$ Edital de Fomento à Pesquisa - Edital 01/2001 - Edital Universal, em 2001.

Destacaram-se como estratégias organizacionais relevantes: a atuação como financiadora dos Programas de Governo; a atuação na modalidade de fomentos de projetos de pesquisa científica e tecnológica; a atuação como agente de fomento ao desenvolvimento empresarial catarinense, a implantação de Incubação de Empresas Catarinenses e de Parques Tecnológicos; a atuação na área de capacitação de pessoal e na melhoria da qualidade das instituições de ensino e pesquisa do Estado.

A classificação proposta por Whittington (2002), sugere que as estratégias podem ser classificadas como Estratégias Sistêmicas, pois, além de serem planejadas, visto que estavam previstas na Lei no 10.355, de 09/01/1997; no Decreto o 1.674, de 12/03/1997 e almejavam objetivos plurais, dentre eles, a capacitação de profissionais e pesquisadores em todos os níveis - pósgraduados, graduados, técnicos e estagiários - a solução de problemas catarinenses como a criação de novas oportunidades de emprego e renda.

4.2.4 Período estratégico IV: Foco na ampliação das modalidades de fomento e na regionalização dos recursos

Em 01 de janeiro de 2003, Luiz Henrique da Silveira é empossado governador do Estado de Santa Catarina, nomeando, para Diretor Geral da FUNCITEC, Antônio Diomário de Queiroz, ex-Reitor da Universidade Federal de Santa Catarina, dando início ao quarto período estratégico do processo de adaptação da instituição. Cinco eventos principais tiveram influência no processo de adaptação estratégica da instituição investigada:

- a alteração da estrutura de poder da FUNCITEC: Diomário de Queiroz assume a Direção Geral da instituição, em 2003;

- entra em vigor o estabelecido no Decreto 307/2003, obrigando que os convênios firmados sejam restritos a entidades com Utilidade Pública Estadual, em 2003;

- criação do Grupo Dominus, em 2004;

- crise financeira no Estado, com contingenciamento do orçamento da FUNCITEC, em 2004; 
- descentralização e regionalização das atividades do Governo, em 2004.

Como estratégias da instituição, destacam-se: a atuação na modalidade de fomento a projetos por meio de Editais de Fomento à Pesquisa Científica; a consolidação das cadeias produtivas no Programa de Arranjos Produtivos Locais; a formação e a capacitação de pessoas e grupos de pesquisa para Ciência, Tecnologia e Inovação; a atuação na Reestruturação do Sistema de Ciência e Tecnologia do Estado e na implantação de Incubadoras de Empresas de Base Tecnológica no Estado.

No modelo sugerido por Whittington (2002), tais estratégias podem ser classificadas como Estratégias Sistêmicas, pois, além de serem planejadas, almejavam objetivos plurais como a capacitação de profissionais, pesquisadores em todos os níveis (pós-graduados, graduados, técnicos e estagiários), a solução de problemas catarinenses e a criação de novas oportunidades de emprego e renda.

\subsubsection{Período estratégico V: Reestruturação, incorporação e crescimento}

No início de 2005, foi aprovada e implementada uma ampla Reforma Administrativa do Governo do Estado. Por meio dessa Reforma, foi unificado e fortalecido o Sistema de Ciência, Tecnologia e Inovação do Estado de Santa Catarina, transformado as duas fundações responsáveis pela pesquisa no Estado - a Fundação de Ciência e Tecnologia (FUNCITEC) e o Fundo Rotativo de Estímulo à Pesquisa Agropecuária (FEPA) - em apenas uma, a Fundação de Apoio à Pesquisa Científica e Tecnológica do Estado de Santa Catarina (FAPESC). Cinco eventos principais influenciaram o processo de adaptação estratégica da instituição investigada, no ano de 2005:

- a transformação da FUNCITEC em Fundação de Apoio à Pesquisa Científica e Tecnológica do Estado de Santa Catarina (FAPESC), incorporando o Fundo Rotativo de Estímulo à Pesquisa Agropecuária (FEPA);

- a adequação da Estrutura Organizacional da FAPESC ao novo Sistema Estadual de Ciência e Tecnologia, criando funções concernentes à área de Pesquisa Agropecuária;

- a mudança na estrutura de poder da FAPESC: assume o cargo de Presidência Rogério Silva Portanova;

- a implementação do Sistema Financeiro de Conta Única no Estado;

- a aprovação do Termo de Outorga que autorizou o contrato direto entre FAPESC e 0 pesquisador.

Destacaram-se como estratégias organizacionais relevantes do período: a atuação como agente fomentador das atividades de Ciência, Tecnologia, Inovação, inclusive da área de Agropecuária, no Estado de Santa Catarina; a atuação como agente de fomento dos Programas de Governo; a atuação na modalidade de fomento de Editais de Pesquisa; a criação do Programa de Gestão da Propriedade Intelectual e a prioridade à área de Meio Ambiente.

$\mathrm{Na}$ classificação de Whittington (2002), as estratégias podem ser classificadas como Estratégias Sistêmicas, pois surgiram de processos deliberados, ou seja, foram previstas no Plano de Governo, no Plurianual e no próprio estatuto da instituição, com objetivos plurais por estarem vinculados a estratégia social do Governo do Estado. 


\section{CONSIDERAÇÕES FINAIS}

Esta investigação procurou descrever e analisar o processo de formação de estratégias na Fundação de Apoio à Pesquisa Científica e Tecnológica do Estado de Santa Catarina (FAPESC), no período compreendido entre 1990 e 2005, considerando quais foram e como se desenvolveram as mudanças na organização, bem como os fatores que as geraram.

A FAPESC é uma organização complexa por ter sua gestão envolvida em questões regulamentares e limitações externas, tais como o que preconiza o poder do Governo do Estado, a legislação que rege a Administração Pública e as aspirações da sociedade quanto ao seu papel e produto. Ao mesmo tempo, observa-se aspectos internos de igual relevância, especialmente os ligados ou decorrentes das diversas instâncias de decisão, dos interesses profissionais e de outros grupos organizados.

De acordo com Whittington (2002), as estratégias relevantes, desenvolvidas pela instituição ao longo do período estudado, são classificadas como Estratégias Sistêmicas, visto que foram pretendidas e planejadas previamente, sem objetivar lucros, pois, na condição de fundação pública, a FAPESC não pode, em nenhuma hipótese, obter lucro sobre seus investimentos.

Como órgão pertencente à administração direta da hierarquia do Governo do Estado, a FAPESC sofreu, em todos os períodos analisados, influência direta das políticas e diretrizes apontadas pelos Planos de Governo.

\section{REFERÊNCIAS:}

ANDREWS, K. R. The concept of corporate strategy. Homewood, Illinois: Down Jones-Irwin, 1971.

ANSOFF, H. I. Implanting strategic management. Englewood Cliffs: Prentice Hall, 1991.

ANSOFF, I. Estratégia empresarial. McGraw-Hill: São Paulo, 1965.

MELLO, R. B. 0 estudo da mudança estratégica organizacional em pequenas empresas de construção de edificações: um caso em Florianópolis. Dissertação (Mestrado em Engenharia de Produção) - Universidade Federal de Santa Catarina, Florianópolis, 1997.

BOWDITCH, J. L.; BUONO, A. F. Elementos do comportamento organizacional. São Paulo: Pioneira, 1997.

CHANDLER, A. Strategy and structure. MIT Press: Cambrige, 1962.

COSTA, L. S. V.; SILVA, J. F. As tipologias estratégicas "realmente" existem? In: Encontro Anual da Associação Nacional dos Programas de Pós-Graduação em Administração. Anais ANPAD: Salvador, 2002.

CUNHA, C. J. C. A. Adaptação estratégica organizacional em ambiente turbulento. (Trabalho apresentado para Concurso de Professor Titular) Programa de Pós-Graduação em Engenharia de Produção - Departamento de Engenharia de Produção e Sistemas. Universidade Federal de Santa Catarina, Florianópolis, 1996. 
De GEUS, A. A empresa viva. IN Harvard Business Review. Estratégias para o crescimento. Rio de Janeiro: Campus, p.95-110, 2000.

GODOY, A. S. Introdução à pesquisa qualitativa e suas possibilidades. Revista de Administração de Empresas. São Paulo, v. 35, n. 2, mar/abr, 1995.

HARDY, C.; FACHIN, R. Gestão estratégica na universidade brasileira: teoria e casos. Porto Alegre: UFRGS. 1996.

IDENBURG, P. J. Four styles of strategy development. Long Range Planning. v.26, n.6,p 132-137, 1993.

KIMBERLY, J. R. Issues in the design longitudinal organizational research. Sociological methods and research, v.4, n.3, 1976.

LIMA. S. M. V. Mudança organizacional: teoria e gestão. Rio de Janeiro: Editora FGV, 2003.

MARIOTTO, F. L. Mobilizing emergent strategies. Relatório nำ10/2000. Núcleo de Pesquisas e Publicações. São Paulo: EAESP/FGV, 2000. (Série Relatórios de Pesquisa).

MINAYO, M. C. S. (Org.). Pesquisa social: teoria, método e criatividade. 19. ed. Petrópolis: Vozes, 1994.

MILES, M. B.; HUBERMAN, A. M. Qualitative data analysis: A source book of new methods. Bervely Hills, C. A.: Sage, 1984.

MINTZBERG, H.; AHLSTRAND, B.; LAMPEL, J. Safári de estratégia Porto Alegre: Bookman, 2000.

MINTZBERG, H.; McHUGH, A. Strategy formation in an adhocracy. Administrative Science Quarterly, v. 30, n. 2, p. 160-197, jun. 1985.

MINTZBERG, H. The strategy concept: Five P's for estrategy. Califórnia Management Review, v. 30, n. 1, 1987.

MINTZBERG, H. An emergent strategy of direct research. Administrative Science Quartely, v.24, p.582-589, 1979.

MINTZBERG, H. Strategy-making in three modes. California Management Review, v.16, n. 2, p. 44$55,1973$.

MINTZBERG, H. Ascensão e queda do planejamento estratégico. Bookman: Porto Alegre, 2004.

MINTZBERG, H.; QUINN, J. Q. O. O processo da estratégia. Porto Alegre: Bookman, 2001.

MINTZBERG, H.; WATERS, J. A. Of Strategies, deliberate and emergent. Strategic Management Journal, 1985.

LINDBLOM, C. E. The science of "muddling through". Public Administration Review. v. 19, p.79-88, 1959.

NICOLAU, I. 0 conceito de estratégia. Instituto de Desenvolvimento da Gestão Empresarial. Lisboa, 2001. 
PETTIGREW, A. M. Contextualist research: a natural way to link theory and practice. In: LAWLER (ed.) Doing research that is use ful in theory and practice. San Francisco: Jossey-Bass, p.222-271, 1985a.

Examining change in the long-term context of culture and politics. In: PENNINGS, J. M. \& Associates. Organizational strategy and change: new views on formulating and implementing strategic decisions. San Francisco: Jossey-Bass, p.269-318, 1985b.

PORTER, M. E. Como as forças competitivas moldam a estratégia. IN MONTGOMERY, C. A.; PORTER, M. Estratégia: a busca da vantagem competitiva. Rio de Janeiro: Campus, p.11-27, 1998.

QUINN, J. B. Strategies for change: logical incrementalism. Sloan Management Review, summer, 1980.

RICHARDSON, R. J. et al. Pesquisa social: métodos e técnicas. São Paulo: Atlas, 1985.

SALAMA, A. O uso da biografia como método de pesquisa para a investigação do desenvolvimento organizacional. Revista de Administração Pública, v. 28, p. 34-42, jan/mar. 1994.

SENGE, P. A Quinta Disciplina: caderno de Campo. São Paulo, Qualitymark, 1996.

STEINER, G. Top management planning. London: The McMillan Company, 1969.

VAN DE VEN, A. H.; POOLE, M. S. Methods for studying innovation development in the Minnesota Innovation Research Program. Organization Science, v.1, n.3, p. 313-335, 1992.

YIN, R. K. Case study research: design and methods. Beverly Hills: Sage, 1990.

WHITTINGTON, R. 0 que é estratégia. São Paulo: Pioneira Thompson, 2002.

\title{
FORMATION OF THE STRATEGY IN CONDITIONS OF RAISED GOVERNAMENTAL INFLUENCE: A LONGITUDINAL ANALYSIS OF FUNDAÇÃO DE APOIO À PESQUISA CINTÍFICA E YECNOLÓGICA DO ESTADO DE SANTA CATARINA - FAPESC
}

\begin{abstract}
This research analyzes the process of strategy formation in the Foundation of Support to the Scientific and Technological Research of the State of Santa Catarina - FAPESC, a body of the government's direct administration structure in the State of Santa Catarina, with the purpose of understanding how its construction occurs: if in a deliberate way, anticipated and rational; or, as an emergency, in consequence of the interactions of the agents present in the organizations' routine. By way of a case study of longitudinal, historical and biographical character, and based in the procedures proposed by the Direct Research (MINTZBERG, 1979; MINTZBERG; McHUGH, 1985), the history of the institution was retrieved in the period comprised between the years of 1990 and 2005. The data has disclosed that, in adapting itself strategically, beyond the predominance of a planned and sistemic strategic behavior, the institution suffered an intense influence from the governmental politics of the State and this, associated with the low power to influence the environment with high environmental determinism, reduced the importance of the management scienter in the success of the company indicating that mechanisms of environmental selection operate to the detriment of the adaptation.
\end{abstract}

Key words: Strategy. Change and adaptation. Formation of the strategy. 